Actas del Seminario Internacional Destinos Turísticos Inteligentes:

nuevos horizontes en la investigación y gestión del turismo

Universidad de Alicante, 26 y 27 de octubre de 2017

\title{
La toma inteligente de decisiones en los procesos de planificación de destinos turísticos de litoral: el plan de infraestructuras turísticas de canarias (PITCAN) ${ }^{1}$
}

\author{
Simancas-Cruz, Moisés \\ Universidad de La Laguna, España \\ Departamento de Geografía e Historia \\ msimancas@ull.es
}

\section{Peñarrubia-Zaragoza, María Pilar \\ Universitat de València Departamento de Geografía M.Pilar.Penarrubia@uv.es}

\section{Rodríguez-Darias, Alberto Jonay}

Universidad de La Laguna Departamento de Antropología jonayalberto@ull.es

Padrón-Ávila, Hugo

Universidad de La Laguna Cátedra de Turismo CajaCanarias-Ashotel-ULL hugopadronavila@gmail.com

Padrón-Candelario, Elísabet

Universidad de La Laguna Cátedra de Turismo CajaCanarias-Ashotel-ULL epadronc@ull.edu.es

Suárez-Perera, David

Universidad de La Laguna Grupo ReinvenTUR

dsuarez@tecnologiasig.com

1. La investigación que da lugar a esta publicación se inscribe en el proyecto titulado «Crisis y reestructuración de los espacios turísticos del litoral español» (CSO2015-64468-P) del Ministerio de Economía y Competitividad (MINECO). Asimismo, constituye uno de los resultados del proyecto «Tecnologías de Información Geográfica aplicadas al análisis y diseño de propuestas innovadoras para la renovación de los alojamientos turísticos», financiado por la Fundación CajaCanarias. 
Actas del Seminario Internacional Destinos Turísticos Inteligentes:

nuevos horizontes en la investigación y gestión del turismo

Universidad de Alicante, 26 y 27 de octubre de 2017

\section{Resumen}

El Plan de Infraestructuras Turísticas de Canarias [PITCAN2017-2013] es un encargo de la Dirección General de Infraestructuras Turísticas de la Consejería de Turismo, Cultura y Deportes del Gobierno de Canarias a un grupo de investigadores de la Cátedra de Turismo CajaCanarias-Ashotel-Universidad de La Laguna y del grupo de investigación de la ULL ReinvenTUR: renovación e innovación turística, a través de la Fundación General de la Universidad de La Laguna. El objetivo del plan es enumerar y priorizar las infraestructuras turísticas públicas a implementar en el período 20172023. Para el desarrollo de este objetivo se ha aplicado un proceso metodológico basado en la participación de agentes clave del destino y la consulta de datos provenientes de diversas fuentes, desarrollando un sistema de indicadores multicriterio y ponderado. El proceso metodológico de identificación, selección, jerarquización, valoración y priorización de las infraestructuras supone la principal aportación de este trabajo. Se trata de una metodología orientada a la gestión inteligente de destinos turísticos.

Palabras clave: criterios de priorización, infraestructuras turísticas, Canarias, PITCAN, toma inteligente de decisiones

\section{Abstract}

The Plan of Tourist Infrastructures of the Canary Islands [PITCAN2017-2013] is a commission of the General Direction of Tourist Infrastructures of the Council of Tourism, Culture and Sports of the Government of the Canary Islands to a group of researchers of the Chair of Tourism CajaCanarias-Ashotel-ULL and the research group of ReinvenTUR: Renovation and Tourist Innovation, through the General Foundation of the University of La Laguna. The goal of the project is listing and prioritizing the public infrastructures to development between 2017 and 2023. The methodology process include a process of participation of different key stakeholders and the consult of data from various sources to design a pondered multicritera indicators system.

Keywords: prioritization criteria, tourist infrastructures, Canary Islands, PITCAN

\section{Introducción}

El desarrollo y aplicación efectiva de instrumentos de planificación y gestión de la actividad turística orientada a minorar sus externalidades negativas y mejorar sus niveles de redistribución económica ha sido una constante durante décadas. Partiendo de la perspectiva del desarrollo sostenible 
Actas del Seminario Internacional Destinos Turísticos Inteligentes:

nuevos horizontes en la investigación y gestión del turismo

Universidad de Alicante, 26 y 27 de octubre de 2017

aplicado a la actividad turística, se pasó por el paradigma de la responsabilidad y, en los últimos años, los destinos inteligentes. Si bien estos tres paradigmas se basan en los mismos pilares (reducción de impactos negativos, ordenación racional de la actividad, mejora de las formas de redistribución de la riqueza, implementación de formas de participación, etc.), existen claras diferencias entre ellos. Así, la sostenibilidad se planteó como la «hoja de ruta» de la planificación y gestión territorial del turismo, aunque de una forma excesivamente orientada a su dimensión ambiental. Por su parte, y de manera muy sintética, el paradigma de la responsabilidad surge con la premisa de hacer más evidente el hecho de que en el sistema turístico participa una amalgama importante de actores interdependientes y parcialmente responsables de los niveles de sostenibilidad de la porción de la actividad turística en la que tienen influencia. De manera que las instituciones públicas se presentan como un agente más en la gestión -sostenible- del destino, si bien con un ámbito de influencia potencialmente relevante.

La perspectiva de los destinos inteligentes surge a partir de las ciudades inteligentes. Éstas últimas, que se ha venido consolidando desde los años noventa, se vertebran en torno a la incorporación de diversas tecnologías en los procesos de planificación y gestión para mejorar su eficiencia y ofrecer a los ciudadanos una mejor calidad de vida. Así, una ciudad puede ser clasificada como inteligente cuando se logra un crecimiento económico sostenible y una alta calidad de vida mediante la inversión en capital humano, un adecuado nivel de participación e infraestructuras que permitan la adecuada difusión de la información a la ciudadanía (Caragliu et al., 2009). El carácter sistémico de la perspectiva de las ciudades inteligentes queda claro a partir del concepto Smart City Wheel, en el que Cohen (2011) integra las dimensiones (1) gobernanza inteligente, (2) medioambiente inteligente, (3) movilidad inteligente, (4) economía inteligente, (5) ciudadanía inteligente y (6) vida inteligente.

La consolidación en los ámbitos académico y técnico de los conceptos ciudades inteligentes, y otros como territorios inteligentes o sistemas inteligentes de gestión, ha motivado la aparición, en los últimos años, de los destinos turísticos inteligentes. En el ámbito académico, esta irrupción ha estado dominada por aportaciones desarrolladas desde el marketing. De esta manera, la mejora de la competitividad del destino y el enriquecimiento de la calidad de la experiencia del turista se presentan como los objetivos 
Actas del Seminario Internacional Destinos Turísticos Inteligentes:

nuevos horizontes en la investigación y gestión del turismo

Universidad de Alicante, 26 y 27 de octubre de 2017

centrales, marcando cierta distancia frente a los objetivos tradicionales de sostenibilidad de los destinos turísticos.

Gran parte de la literatura académica sobre destinos turísticos inteligentes obvia la perspectiva integradora que se refería anteriormente respecto a las ciudades inteligentes y centra la atención en el uso de tecnología, presentándola en ocasiones como un fin en sí mismo (y no como un medio para la mejora del sistema turístico en su conjunto). Ello está determinando que los destinos turísticos inteligentes se basen en el internet de las cosas, computación en la nube y servicios online para turistas (Buhalis y Amaranggana, 2014 y 2015; Wang, Li y Yunpeng, 2013). El calificativo inteligente parece haberse convertido en un eslogan para productos y servicios que incorporan tecnologías de la información y comunicación (Boes, Buhalis y Inversini, 2015). En este sentido, 'lo inteligente' se ha convertido en una especie de mantra publicitario que ha venido a sustituir a la ola de productos y servicios «2.0» que comenzó a inundar el panorama turístico hace aproximadamente una década.

De esta manera, el modelo de destinos turísticos inteligentes ha emergido con fuerza como nueva referencia para los procesos de planificación y gestión territorial de las áreas turísticas. Sin embargo, la banalización del término, sus perfiles conceptuales difusos, las diferentes interpretaciones, la tardanza en aportar resultados prácticos y, sobre todo, su centralización instrumental en cuestiones vinculadas fundamentalmente a la tecnología, constituyen algunos factores que hacen que cuestionemos su eficiencia. En este sentido, se plantea que lo esencial del concepto es la toma inteligente de decisiones para que los destinos turísticos se inserten en el paradigma de la sostenibilidad y la competitividad territorial.

Del mismo modo, se considera que la conceptualización de los destinos inteligentes debe recoger el carácter sistémico recogido en las ciudades inteligentes. De modo que no se trata únicamente de un desafío técnico, sino, más bien, de un cambio organizativo orientado a la innovación, la participación, el manejo de diversas variables y gran cantidad de datos y la comunicación. Se trata de recuperar la filosofía del paradigma de la sostenibilidad, pero incorporando cuatro importantes novedades, que constituyen los elementos estructurales de la gestión inteligente de los destinos turísticos: la consideración integral de sus cuatro dimensiones (ambiental, económica, social e institucional), la toma de decisiones a partir de una ingente cantidad de datos (big data), la percepción del cliente (turista), así como la 
Actas del Seminario Internacional Destinos Turísticos Inteligentes:

nuevos horizontes en la investigación y gestión del turismo

Universidad de Alicante, 26 y 27 de octubre de 2017

participación de los agentes, en un intento de lograr procesos efectivos de gobernanza participativa.

En este trabajo se expone el proceso metodológico seguido para la elaboración del Plan de Infraestructuras Turísticas de Canarias [PITCAN2017-2013]. Es un encargo de la Dirección General de Infraestructuras Turísticas de la Consejería de Turismo, Cultura y Deportes del Gobierno de Canarias a un grupo de investigadores de la Cátedra de Turismo CajaCanarias-Ashotel-Universidad de La Laguna y del grupo de investigación de la ULL ReinvenTUR: renovación e innovación turística, a través de la Fundación General de la Universidad de La Laguna. Se trata de una herramienta estratégica orientada a la gestión inteligente de destinos turísticos para la implantación de elementos que permitan el desarrollo adecuado de actividades turísticas. Así, este trabajo muestra un caso de interés sobre los procesos de adaptación de los destinos a las formas inteligentes de gestión.

Se pretende que el PITCAN contemple el conjunto de expectativas realistas y específicas que los diferentes actores clave del sistema turístico canario pretenden conseguir en materia de infraestructuras turísticas a escala regional, insular y municipal. Para ello, el PITCAN se ha planteado como un programa, a modo de base de datos abierta y sistemática de infraestructuras, que ha resultado de un proceso de identificación participativa y de priorización objetiva y sintética en función de productos turísticos, perfiles del turista (clientes), así como déficits de equipamientos y dotaciones existentes en los espacios turísticos. Por ello se ha planteado como una sistematización propositiva de infraestructuras - no proyectos de obras o actuaciones- con una especificación (jerarquización, valoración y priorización) de su importancia estratégica. Ello lo aproxima al concepto de inteligencia territorial, entendida como la aplicación de técnicas y procedimientos propios de la «inteligencia competitiva» para el inventario, diagnóstico, valoración y difusión de la información orientada a la mejora, sostenibilidad y competitividad de un territorio.

\section{Premisas básicas que han guiado la elaboración del PITCAN}

El objetivo principal del PITCAN es elaborar una base de datos con las infraestructuras turísticas estratégicas. No se plantea como una lista de demandas en materia de infraestructuras, sino como una relación de actuaciones priorizadas. Ésta ha resultado de un proceso de identificación participativa de los déficits de equipamientos y dotaciones existentes en los espacios turísticos 
Actas del Seminario Internacional Destinos Turísticos Inteligentes:

nuevos horizontes en la investigación y gestión del turismo

Universidad de Alicante, 26 y 27 de octubre de 2017

$\rightarrow$ la propuesta de infraestructuras $\rightarrow$ la determinación objetiva y sintética de su relevancia estratégica en función de productos turísticos, perfiles del turista (clientes), etc. $\rightarrow$ la identificación de las actuaciones prioritarias. Los objetivos específicos son los siguientes:

- Dotar a las infraestructuras que deben servir de soporte al desarrollo de productos turísticos de una verdadera dimensión turística y territorial. Se trata de adecuar las infraestructuras a los productos que van a articular los destinos/áreas turísticas, y, por tanto, a su modelo de negocio.

- Generar un proceso participativo para la elaboración del diagnóstico de los déficits de infraestructuras turísticas y de definición de las prioridades a través de la implicación activa de los/as responsables públicos y técnicos de diversas instituciones. Así, el PITCAN ha clarificado los intereses y las posturas de los agentes implicados a escala insular y municipal, jerarquizado y priorizado de forma creativa y objetiva las infraestructuras turísticas, así como ha promocionado el diálogo y la reflexión en esta materia.

- Elaborar una base de datos abierta, dinámica y flexible de infraestructuras turísticas. EI PITCAN se ha planteado como un proceso, por lo que la base de datos tiene una clara vocación no finalista, susceptible de una constante revisión en función de las prioridades y posibles líneas de financiación.

Para ello, el equipo redactor del PITCAN partió de cuatro premisas esenciales.

a) La isla como el espacio turístico. Las infraestructuras contempladas en el PITCAN se plantean desde el diseño de una estrategia integral del correspondiente espacio turístico. Se trata de evitar caer en el tradicional reduccionismo de suponer que el espacio turístico se limita a lo urbanizado por y para el turismo y, por tanto, sólo al espacio urbano-turístico, donde se concentra principalmente el alojamiento turístico y los equipamientos de servicio, aprovechando procesos de economía por aglomeración. Por tanto, el PITCAN entiende como espacio turístico no sólo donde se alojan los turistas, sino la totalidad de cada isla del Archipiélago canario. Para lograr lo anterior, el PITCAN formula una idea del territorio turístico. Esta relaciona las 
Actas del Seminario Internacional Destinos Turísticos Inteligentes:

nuevos horizontes en la investigación y gestión del turismo

Universidad de Alicante, 26 y 27 de octubre de 2017

áreas turísticas de litoral (el 1,76\% del espacio turístico de Canarias) con el contexto territorial donde se ubican los recursos turísticos (el resto de la superficie del Archipiélago). Así, se parte de la premisa de que cada isla presenta una relación entre nodos de partida (las áreas turísticas), en los que se concentran las pernoctaciones, y los "puntos de interés turístico», entendidos como lugares de visita. De esta manera, se genera un modelo territorial turístico, en el que la suma de nodos de concentración turística y puntos de interés turístico constituyen, en su conjunto, un sistema territorial-turístico en el que instalar las infraestructuras turísticas.

b) El plan como un inventario abierto, dinámico, flexible y en construcción de infraestructuras turísticas insertado en un proceso de toma inteligente de decisiones. EI PITCAN no se plantea como un documento finalista o definitivo. El objetivo es que la base de datos del PITCAN tenga la capacidad de adaptación para transformarse y dar respuesta a los cambios institucionales y políticos, fuentes de financiación y demandas sectoriales en materia de infraestructuras turísticas. Por tanto, pretende dar respuesta a las novedades y necesidades que van surgiendo en materia de infraestructuras turísticas, incorporando de un modo sistemático todos los elementos que puedan ser útiles y enriquecedores para el destino, en función de las oportunidades y fuentes de financiación.

c) La necesidad de otorgar una dimensión turística y estratégica a las infraestructuras. Para ello se ha partido de la consideración del producto turístico. Se ha estimado que cada destino turístico -a escala insular, municipal o local (área turística o microdestino)- está formado por un conjunto de elementos que tienen la capacidad de atraer al consumidor. La diversidad de productos que pueden desarrollarse explica una multiplicidad de formas de consumirlos, teniendo todos ellos en común la necesaria localización en un territorio, dada la necesidad de su uso y disfrute in situ. Ello ofrece una gran variedad de servicios para atraer a un determinado segmento de mercado, que se sentirá motivado por el tipo de actividades vinculadas al producto turístico desarrollado en el lugar.

En relación con lo anterior, se planteó la conveniencia de adecuar la demanda institucional de infraestructuras al turista (el cliente). Se ha planteado esencial la optimización de las infraestructuras según 
Actas del Seminario Internacional Destinos Turísticos Inteligentes: nuevos horizontes en la investigación y gestión del turismo

Universidad de Alicante, 26 y 27 de octubre de 2017

las necesidades del cliente. Para ello se ha partido de nuestros perfiles de turistas. Para ello, el PITCAN parte de la importancia del análisis de datos para identificar y priorizar las infraestructuras turísticas de manera inteligente y adaptado a la segmentación y necesidades del visitante; se trata de «transformar datos (información) en conocimiento».

Del mismo modo, se ha valorada que las infraestructuras propuestas se inserten en una estrategia turística. Se procurará que deriven del diagnóstico (problemas y oportunidades) y objetivos-aspiraciones-propuestas, estrechamente relacionadas con el momento y el lugar en que se implemente, las necesidades reales y la disponibilidad de financiación.

d) La necesaria participación de los actores en la identificación y priorización de las infraestructuras turísticas. El alejamiento del «habitual modelo burocrático y de control jerárquico, caracterizado por imposiciones descendentes (top-down)" ha determinado que la elaboración del PITCAN se ha planteado como un proceso metodológico de toma de decisiones inteligente, estratégica y global en materia de planificación de infraestructuras turísticas. Para ello, el PITCAN ha articulado en la opinión de los actores (técnicos, responsables públicos, empresariales, sociales, etc.) competentes en la toma de decisiones en materia de infraestructuras turísticas públicas. De esta manera, en lugar de que el protagonismo de su elaboración se asuma unilateralmente, se ha optado por la generación de un elevado número de interacciones, el incremento de las referencias, el conocimiento y las implicaciones personales, las relaciones de responsabilidad con el Plan, así como de oportunidades de aprendizaje para detectar los déficits y priorizar las infraestructuras desde la perspectiva de una estrategia turística.

e) Las infraestructuras constituyen elementos básicos e imprescindibles para el diseño y desarrollo de experiencias y productos turísticos. Las infraestructuras sustentan una serie de productos que tratan de cubrir las expectativas de los turistas. Así, recurriendo, de nuevo, al lenguaje informático: mientras que las infraestructuras turísticas serían el hardware, el producto turístico sería el software. 
Actas del Seminario Internacional Destinos Turísticos Inteligentes:

nuevos horizontes en la investigación y gestión del turismo

Universidad de Alicante, 26 y 27 de octubre de 2017

2.1. La metodología del PITCAN: la identificación y jerarquización inteligente de infraestructuras turísticas

EI PITCAN se ha planteado como un programa, a modo de base de datos abierta y sistemática de infraestructuras turísticas. Así, se ha planteado como una sistematización propositiva de infraestructuras -no proyectos de obras o actuaciones- con una especificación (jerarquización, valoración y priorización) de su importancia estratégica. De esta manera, se desarrolla a escala de plan o programa, no bajando a la escala de proyectos, cuya competencia corresponde a las entidades proponentes.

El proceso metodológico de identificación, selección, jerarquización, valoración y priorización de las infraestructuras supone la principal aportación de este trabajo. Como se ha referido, se presenta como una metodología orientada a la gestión inteligente de destinos turísticos a partir de los datos disponibles y los agentes accesibles (figura 1 ).

Figura 1. Metodología de identificación y priorización de infraestructuras turísticas
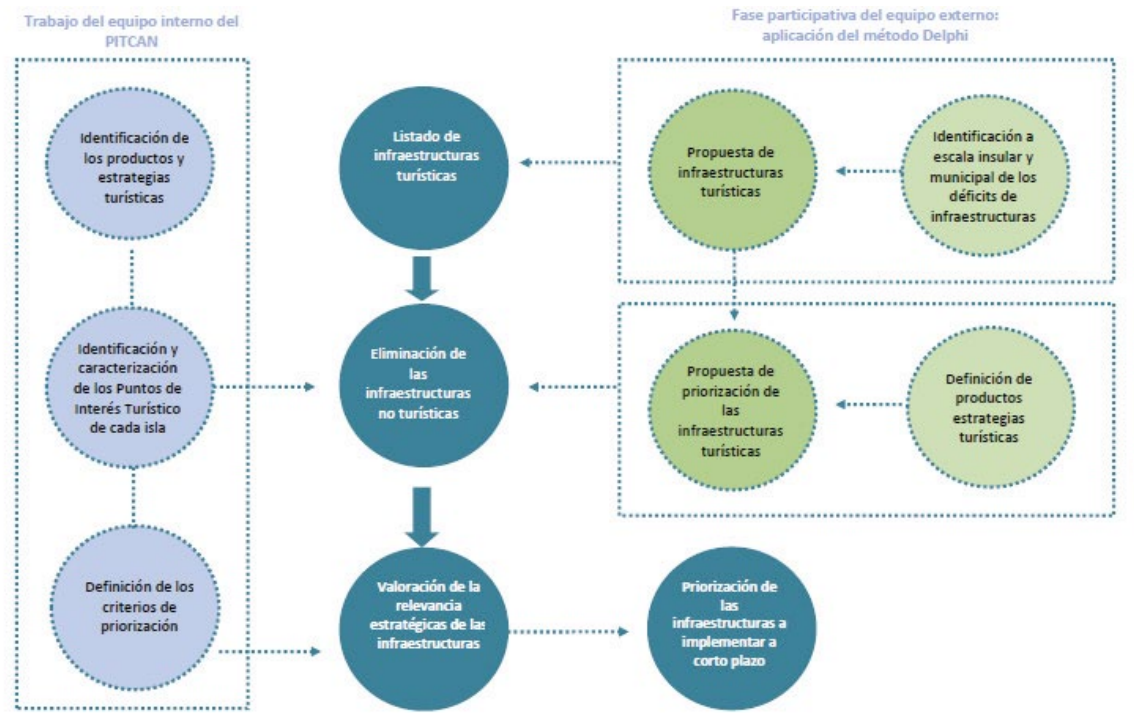
Actas del Seminario Internacional Destinos Turísticos Inteligentes:

nuevos horizontes en la investigación y gestión del turismo

Universidad de Alicante, 26 y 27 de octubre de 2017

Primera fase. Identificación de las infraestructuras

La primera fase del proyecto consistió en la identificación de las infraestructuras turísticas necesarias para el desarrollo de productos turísticos clave en el archipiélago. Para el desarrollo de esta fase se diseñó una metodología de trabajo con técnicos responsables del Gobierno de Canarias, los siete Cabildos Insulares, los 88 ayuntamientos de Canarias, gerentes de las asociaciones turísticas y representantes de la sociedad civil organizada. A partir de ese momento se empezó un proceso en dos escalas geográficas de trabajo: la insular y la municipal.

- A escala insular. Una vez seleccionados los agentes que compondrán el panel de expertos, se procede al envío de un primer cuestionario de respuestas abiertas con el objeto de obtener información sobre cuáles son los productos turísticos que estos agentes consideran especialmente relevantes para cada área turística, así como la disponibilidad y adecuación de las infraestructuras necesarias para su desarrollo. Los datos obtenidos sirvieron de base para el segundo cuestionario. Este contuvo respuestas cerradas, con el fin de concretar con mayor detalle las percepciones de los agentes de los paneles en cuanto a las infraestructuras turísticas necesarias para el desarrollo de los productos turísticos que articulaban o pretendían que articulasen su estrategia turística se pretendían las áreas turísticas y su priorización. Tras este segundo cuestionario, de acuerdo con la metodología Delphi, se procedió a enviar un tercer cuestionario, en el que se debía priorizar las infraestructuras propuestas. Durante el desarrollo de las fases referidas, se desarrollaron reuniones individuales y en grupo, en los que se compartieron la metodología y los resultados obtenidos. Del mismo modo, la exposición del proceso en diversos foros y en la Consejo Canario de Turismo resultó relevante.

- A escala municipal. Si bien inicialmente se pretendía reducir la consulta a los 15 municipios «esencialmente» turísticos, la premisa de que cada isla constituye en sí mismo el destino turístico, determinó que ésta se ampliase a la totalidad de entidades municipales. La Federación Canaria de Municipios desempeñó un papel esencial en la labor de contacto con los técnicos responsables de los mismos. En este caso, se decidió sólo enviar el tercer cuestionario, en cuanto 
Actas del Seminario Internacional Destinos Turísticos Inteligentes:

nuevos horizontes en la investigación y gestión del turismo

Universidad de Alicante, 26 y 27 de octubre de 2017

aglutina la identificación y priorización de las infraestructuras turísticas.

Se obtuvo respuesta de 37 entidades: el Gobierno de Canarias, Promotur, los siete Cabildos Insulares, 24 municipios (el 27\%), la Asociación empresarios turísticos de Fuerteventura, una entidad privada (Gema Arquitectos) y el grupo de investigación universitario del Patrimonio (Departamento de Construcción Arquitectónica de la Universidad de Las Palmas de Gran Canaria). Asimismo, Gestión y Planeamiento Territorial y Medioambiental, S.A (Gesplan) se ha incorporado, en cuanto es la empresa pública encargada de los Planes de Modernización, Mejora e Incremento de la Competitividad.

Como consecuencia fundamentalmente de las propuestas de los Cabidos Insulares, las infraestructuras turísticas propuestas se ubican en 76 de los 87 municipios de Canarias (el 86\%). Superando claramente el contexto territorial de los municipios considerados "turísticos» (aquellos que fundamentalmente albergan la infraestructura alojativa), desde una perspectiva más amplia del espacio turístico como aquellas áreas en las que se desarrolla la práctica de actividades turísticas. No en vano el turista emplea el territorio como soporte, pero también como recurso, así como el escenario donde desarrolla sus experiencias. Así, el espacio turístico es «[...] el territorio donde se localizan los elementos que están relacionados con la actividad turística: los recursos que pueden ser aprovechados para formar productos turísticos, las infraestructuras de acceso y apoyo, los alojamientos y el espacio de las actividades» (Vera, López, Marchena y Antón, 2011: 83).

Tras este proceso, se procedió a desestimar aquéllas que carecían de una dimensión turística. Como resultado se obtuvo una base de datos con 652 infraestructuras propuestas por diferentes agentes de las islas.

Segunda fase. Priorización

La priorización de las infraestructuras propuestas supuso un reto importante con potenciales implicaciones sociales, económicas y políticas. La pregunta de base es ¿cuáles de las infraestructuras turísticas propuestas son más razonables o prioritarias desde el punto de vista de la estrategia turística de cada espacio insular y, por tanto, coherentes con la lectura de su entorno, los productos turísticos a desarrollar y las demandas de los diferentes actores del sistema turístico? Desde este planteamiento, la especificación (valoración) 
Actas del Seminario Internacional Destinos Turísticos Inteligentes: nuevos horizontes en la investigación y gestión del turismo

Universidad de Alicante, 26 y 27 de octubre de 2017

de la importancia estratégica de las infraestructuras turísticas pretende definir un orden jerárquico a través de unos valores numéricos totales.

Lo anterior determinó necesaria optar por una metodología que combine las distintas dimensiones, objetivos, actores y escalas que se hallan envueltos en el proceso de toma de decisiones, sin sacrificar la calidad, confiabilidad y consenso en los resultados. Por ello se ha recurrido a una metodología multicriterio, en cuanto posibilita contemplar la diversidad de factores a integrar en el proceso de determinación de la importancia estratégica de las infraestructuras turísticas, y, sobre todo, transformar las mediciones y percepciones en una escala única, de modo de poder comparar los elementos y establecer órdenes de prioridad. A continuación, se detallan los diferentes criterios tenidos en cuenta.

Criterio 1.1. Caracterización del perfil del turista: adecuación de la infraestructura propuesta a la estrategia de marketing insular. El conocimiento de los turistas que hacen uso de los espacios turísticos, entendidos como aquellos con motivaciones múltiples que buscan diferentes experiencias según el momento y el contexto específico, ha resultado una labor clave para la identificación de déficit de infraestructuras y la determinación de su importancia estratégica. El análisis de la segmentación del mercado nos ha permitido identificar y caracterizar las características de la demanda, cuyos gustos y necesidades se corresponderán con ámbitos turísticos concretos. El objetivo de este análisis es determinar, aplicando un criterio cuantitativo, la adecuación de la infraestructura propuesta a la tipología de cliente hacia la que se desea orientar la actividad turística, definida en cada plan de marketing insular.

La caracterización del cliente se inició considerando las nueve categorías "meta» contempladas en el Plan de Marketing de la marca Islas Canarias para el año 2016, elaborado por Promotur Turismo Canarias, entendiendo por tal al conjunto de productos que resuelven una misma necesidad o deseo de los potenciales clientes. Estas categorías parten de la relación realizada por el Instituto de Turismo de España durante la elaboración de su Plan de Estratégico de Marketing 2011-2013, y determina las categorías en la que los destinos turísticos españoles cuentan con mayor potencial competitivo sirviendo como base para la identificación de las categorías meta de la marca Islas Canarias. 
Actas del Seminario Internacional Destinos Turísticos Inteligentes:

nuevos horizontes en la investigación y gestión del turismo

Universidad de Alicante, 26 y 27 de octubre de 2017

El análisis de las diferentes infraestructuras turísticas propuestas determinó la necesidad de incorporar otras categorías, al detectarse que, algunas de ellas no podían ser incluidas en las elegidas como «meta»; así, se decidió rescatar la categoría potencial de "Arte y cultura». A su vez, la categoría de sol y playa se le ha intentado otorgar un nuevo enfoque, al tratarse de la categoría predominante, ampliando la oferta complementaria dirigida al disfrute de cada uno de los destinos insulares, con el fin de llegar a un mayor número y variedad de turistas; ello explica que concentre casi la totalidad de las actividades que pueden realizarse en todas las islas, así como que la mayoría de las infraestructuras turísticas propuestas puedan adecuarse a esta categoría. Asimismo, cada una de las categorías conlleva una serie de actividades propias.

Partiendo como marco general de estas categorías, cada una de las islas ha establecido sus propias tipologías de turista, así como la conveniencia de una serie de actividades propias para cada territorio. Estas están contenidas en los diferentes documentos: «Análisis 2016 y Plan de Acciones 2017» elaborado por el Patronato de Turismo de Fuerteventura, «El Plan estratégico Gran Canaria Turismo 2020» por el Patronato de Turismo de Gran, el «Plan promocional y turístico 2017» de Turismo de Lanzarote y la Estrategia Turística de Tenerife (2017-2020/2030» de Turismo de Tenerife, así como el «Plan Director de Turismo 2012-2015» del Cabildo de La Gomera. En la medida en que El Hierro y La Palma no disponen de tal documento, dada su consideración de «islas verdes», se ha decidido seguir los criterios de segmentación de La Gomera. Como se puede apreciar, algunas de las categorías definidas por cada isla no se encuentran recogidas en el Plan de Marketing de las Islas Canarias. Al mismo tiempo, cada plan suele emplear directamente las actividades para especificar la tipología de público objetivo al que se refiere la estrategia. Del mismo modo, lo que hacen, en su mayoría, es combinar actividades concretas con tipologías de turistas, más que establecer propiamente categorías. Con el fin de unificar criterios para establecer la relación posible entre la infraestructura propuesta y la categoría o tipología de cliente propuesta para cada isla, se ha elaborado una tabla de correspondencias entre las categorías propuestas, las actividades que permite cada una de ellas, así como las infraestructuras que podrían ser justificadas desde la estrategia de marketing en cuanto a la adaptación de tipología de cliente deseada.

Del mismo modo, en la calificación de las infraestructuras se han considerado dos categorías adicionales denominadas "soporte general» y 
Actas del Seminario Internacional Destinos Turísticos Inteligentes:

nuevos horizontes en la investigación y gestión del turismo

Universidad de Alicante, 26 y 27 de octubre de 2017

"soporte específico»: mientras las primeras hacen referencia a aquéllas que dan apoyo a la totalidad de categorías existentes en cada isla, de modo que tienen una afectación territorial y sectorial transversal a la totalidad del ámbito geográfico, las segundas tienen un impacto local y limitada a la actividad turística. Al mismo tiempo, existen propuestas, como los termómetros urbanos planteados por Promotur Turismo de Canarias, que se proyectan como elementos susceptibles de ser incorporados simultáneamente a otras infraestructuras turísticas.

Finalmente se ha asignado un valor dependiendo de si la infraestructura se adaptaba a las categorías propuestas por la estrategia de marketing de cada isla, o si no lo hacía. Si no se adaptaba, su valor para este criterio seria 0 , mientras que si se adaptaba dependería su valor de la cantidad de actividades que permita realizar, valorando con un máximo de 15 puntos aquellas que permitan hasta 5 actividades o aquellas que, por su carácter general, se identifiquen como de soporte general. El valor otorgado es máximo para las infraestructuras generales (transversales) y mínimo para las específicas.

\section{Criterio 1.2. Importancia de las infraestructuras para el modelo turístico} insular: A partir de datos provenientes de la Encuesta de Gasto Turístico de Canarias de 2016, se ha procedido a cuantificar y hacer una aproximación a las características de los turistas en las islas. Se han seleccionado una serie de variables que se consideran relevantes al permitir conocer la adecuación del cliente con la estrategia propuesta en relación con el gasto realizado por los turistas, su perfil sociodemográfico, así como las características generales de su viaje. Ello ha permitido la comparación de resultados entre los principales municipios turísticos de las islas. Para ello se han seleccionado una serie de variables que se consideran relevantes al permitir conocer la adecuación del cliente con la estrategia propuesta:

Aspectos que influyen en la elección: Clima/sol, Playas, Tranquilidad/descanso/ Relax, Paisajes, Lugar adecuado para niños, Oferta cultural, Calidad entorno ambiental, Oferta Turismo activo, Ambiente nocturno/ Diversión, Parques de ocio, Compras, Actividades náuticas. En la encuesta los indicadores son seleccionados por el turista en función de su prioridad permitiendo la multi-respuesta.

Indicadores de impresión y satisfacción con el destino (Valoración de aspectos y servicios): zonas de baño, paisajes, calidad del medio ambiente, 
Actas del Seminario Internacional Destinos Turísticos Inteligentes:

nuevos horizontes en la investigación y gestión del turismo

Universidad de Alicante, 26 y 27 de octubre de 2017

actividades culturales, actividades deportivas, golf, parques de ocio, ambiente nocturno / diversión, diversión / recreo para niños.

La valoración de cada uno de los datos se ha efectuado en relación al valor medio de cada variable para el total de las Islas Canarias. De esta manera, cada dato relativo a cada indicador cuyo valor sea superior a la media de ese mismo indicador para la región canaria, se le otorga un punto, mientras que los que presentan un valor inferior a la media obtienen un cero. La calificación final se compondrá de la suma de puntos siempre y cuando los valores analizados tengan que ver con las categorías establecidas para cada infraestructura acorde con la matriz.

\section{Criterio 1.3 Afluencia de visitantes y evaluación del impacto competitivo:} el análisis de los «lugares de interés turístico» ha permitido determinar si los sitios propuestos en los que ubicar determinadas infraestructuras turísticas cuentan con una afluencia significativa de visitantes y si aquélla es capaz de solventar los problemas principales señalados por los visitantes de la zona. Además, se pretende que el análisis ayude a medir y clasificar el impacto de una infraestructura en el área en la que se adscribe. Para su análisis se ha recurrido a los datos estadísticos oficiales $y$, sobre todo, a dos fuentes:

- La afluencia de visitantes a los lugares mediante los «heatmap» (o 'mapa de calor') de InstaSights. Como es sabido, recoge las fotos con coordenadas subidas a la red social Instagram, mediante el uso de un código de colores. Así, permite identificar las zonas más fotografiadas. En la base de datos se han utilizado valores entre 0 (ínfima o nula) y 5 (máxima) para reemplazar al código de colores que es empleado por esta plataforma para la categorización de los lugares.

- Los comentarios, recomendaciones, valoraciones y quejas contemplados en la plataforma Tripadvisor. Su consideración ha permitido incorporar la percepción de los visitantes al PITCAN, y, sobre todo, determinar si las infraestructuras propuestas se ajustan a sus demandas. En la base de datos se ha creado la casilla «Interrelación con comentarios», en la que se ha expuesto si la medida propuesta por la administración pública se orienta a dar solución o reducir las quejas señaladas por los usuarios del lugar: en caso afirmativo, se indica en esta casilla "Se adapta a las necesidades»; por el contrario, cuando las medidas de mejora no se corresponden con los problemas 
Actas del Seminario Internacional Destinos Turísticos Inteligentes:

nuevos horizontes en la investigación y gestión del turismo

Universidad de Alicante, 26 y 27 de octubre de 2017

reflejados por los visitantes, se comenta el objetivo perseguido por las actuaciones en cada lugar.

Tras la recopilación de esta información, se ha creado la categoría «tipo de mejora» que hace referencia a la ventaja competitiva que obtendría el lugar visitado si se implantase una determinada infraestructura: competitividad, diversificación, redistribución de flujos, especialización, sostenibilidad y nulo. Para la categorización de las mejoras se ha tenido en cuenta la afluencia de visitantes al lugar en el que se ubican las infraestructuras, el tipo de producto que se desarrollaría a través de las mismas y el objetivo principal perseguido mediante su desarrollo. Se han diferenciado un total de seis tipos posibles:

- Competitividad: Fomento del atractivo del producto turístico principal de la isla en aquellos lugares con un importante flujo de visitantes o en zonas distantes a los núcleos poblacionales. La infraestructura pretende mejorar la experiencia del mismo tipo de visitantes en las áreas turísticas tradicionales.

- Diversificación: Desarrollo de productos turísticos menos explotados en la isla o innovadores. El objetivo es la atracción de nuevos segmentos turísticos.

- Redistribución de flujos: Desarrollo de productos turísticos innovadores o tradicionales para el destino en áreas cercanas a núcleos poblacionales poco desarrollados turísticamente. La intención es fomentar la dispersión de los turistas por el destino y la generación de rentas por turismo en lugares diferentes a los tradicionales.

- Especialización: Fomentar el desarrollo de uno de los productos turísticos principales de la isla, el cual supone una ventaja competitiva para la misma al diferenciarla de los productos turísticos principales que son explotados en otras islas.

- Sostenibilidad: Medidas relacionadas con este paradigma de desarrollo.

- Nulo: Infraestructuras enfocadas principalmente a la población local y con escaso impacto en los visitantes del destino turístico.

La utilización de este análisis como herramienta de priorización de las infraestructuras turísticas ha supuesto una parametrización de ambos indicadores. Así, al análisis de la afluencia se le ha otorgado una puntuación entre cero y cinco puntos, teniendo en cuenta la categorización numérica previamente 
Actas del Seminario Internacional Destinos Turísticos Inteligentes:

nuevos horizontes en la investigación y gestión del turismo

Universidad de Alicante, 26 y 27 de octubre de 2017

realizada. La relación entre infraestructuras propuestas con los comentarios en TripAdvisor se ha valorado con cero puntos si no se relacionaban o con dos puntos si la realización de la infraestructura se ajustaba a las demandas de turistas.

Criterio 1.4 La medición del impacto económico de la implantación de la infraestructura turística: la medición de la intensidad del impacto económico que generaría la mejora perseguida por la infraestructura turística en la zona («Impacto en el área») se ha estimado utilizando la graduación de "bajo», "medio» y "alto». Ésta se ha realizado teniendo en cuenta seis aspectos:

- Nuevas áreas turísticas. La creación de pequeños atractivos en zonas con una baja afluencia de visitantes permite redistribuir las rentas generadas por la actividad, fomentar el crecimiento del empleo en la zona y evitar la masificación de áreas turísticas tradicionales.

- Fomento del tiempo de estancia. Ciertas infraestructuras incitan a los visitantes a permanecer durante períodos más largos en la zona, lo cual incrementa las posibilidades de que consuman en el lugar y de que compartan su experiencia a través de redes sociales.

- Fomento del gasto en los alrededores. Determinadas acciones, como el embellecimiento de las calles, mejoran la experiencia de los visitantes, pero no los incita a gastar en la zona. Sin embargo, la creación de paseos marítimos fomenta la aparición de negocios alrededor de los mismos y, con ello, el consumo por parte de los turistas.

- Nuevos productos o servicios para el destino: La diversificación productiva fomenta la atracción de nuevos segmentos turísticos y la mejora de la experiencia de los visitantes tradicionales al apreciar el destino como una entidad con multitud de actividades para desarrollar. Además, se fomenta la puesta en marcha de negocios por parte de la población local que estén relacionados con estos productos.

- Interés actualmente suscitado por la zona. En los lugares altamente frecuentados, el impacto de las infraestructuras turísticas tenderá a ser menor, al igual que en aquellos poco o nada conocidos por los visitantes, las celdas de color negro, blanco (0), rojo (4) y carmesí (5) de InstaSights. Sin embargo, en lugares conocidos, pero poco frecuentados, el impacto de las infraestructuras tenderá a ser mucho mayor, las celdas de color verde (1), amarillo (2) y naranja (3). 
Actas del Seminario Internacional Destinos Turísticos Inteligentes:

nuevos horizontes en la investigación y gestión del turismo

Universidad de Alicante, 26 y 27 de octubre de 2017

El análisis del impacto económico esperado tras la instalación de la infraestructura turística propuesta se ha valorado como -1 si aquélla no se relaciona directamente con la actividad turística, cero puntos si el impacto esperado es bajo, 1,5 si se espera que su efecto es medio y 3 puntos si es alto. Los puntos de las infraestructuras que no han podido ubicarse a través de InstaSights han pasado a formar parte de los otorgados por el análisis del impacto económico esperado, resultando -1 puntos para aquellas actuaciones que no resultan necesarias para la actividad turística, 2 puntos para las que poseen un impacto bajo, y 5 y 8 puntos para las que tienen un impacto medio y alto, respectivamente.

Finalmente, dentro de este punto también se ha integrado una valoración cualitativa del equipo interno de la importancia estratégica de la infraestructura propuesta. Para ello se ha considerado la justificación expresada en el tercer cuestionario por parte de las entidades proponentes.

Criterio 2. Criterios de los responsables públicos: el fin último del PITCAN de generar un proceso participativo para la elaboración del diagnóstico de los déficits de infraestructuras turísticas y, sobre todo, su priorización, determina que la implicación activa de los/as responsables públicos y técnicos de diversas instituciones resultase esencial. Para ello, desde la premisa de que una forma de reforzar el sentimiento de responsabilidad común, sea la generación de un proceso participativo, el PITCAN ha incorporado a los actores estratégicos no sólo a los procesos de diagnóstico de los déficits de infraestructuras turísticas, sino también en los momentos discursivos, deliberativos, decisorios y de gestión, en concreto, en la labor de su identificación y, sobre todo, priorización. Ello ha permitido recoger su percepción de la relevancia estratégica de los actores claves implicados, y, por tanto, sus necesidades, expectativas y aspiraciones.

Este enfoque participativo no sólo ha posibilitado que el documento resultante dote de visibilidad y difusión al proceso, sino que también proporcione las observaciones y opiniones -saber-, procedimientos -saber hacer- $y$ actitudes - saber ser- acerca de las necesidades en materia de infraestructuras turísticas. Así, durante su proceso de elaboración se ha procedido a escuchar, recoger y concordar los distintos intereses, deseos y objetivos que expresan los diversos agentes que intervienen en la identificación y priorización de las infraestructuras turísticas. Este carácter abierto y participativo del proceso a veces ha conllevado la inclusión de acciones de 
Actas del Seminario Internacional Destinos Turísticos Inteligentes:

nuevos horizontes en la investigación y gestión del turismo

Universidad de Alicante, 26 y 27 de octubre de 2017

condición generalista o expresiones de buena voluntad que pueden no traducirse en una infraestructura concreta o con una clara dimensión turística.

Criterio 3. Infraestructura contemplada en un plan: como ya se ha comentado, se ha intentado que el PITCAN no sea una mera colección de intervenciones (obras) sectoriales, cartesianas, sin articulación con la planificación territorial y el planeamiento urbanístico. Lo mismo sucede con actuaciones escasamente adaptadas a la idiosincrasia del espacio turístico, puntuales, inconexas y aisladas, que buscan soluciones paliativas a las "partes» y problemas puntuales del espacio turístico o simplemente a su modernización, o planteamiento espontáneo o coyunturalmente desde la oportunidad de existencia de fuente de financiación o de las exigencias de las administraciones municipales para suplir sus déficits de servicios e infraestructuras públicas.

Esta vocación determinó que entre los criterios de priorización se pondere que las infraestructuras turísticas propuestas estén contempladas en cualquier plan o programa, integral o sectorial (criterio 3). Ello implica que está integrada en una acción estratégica.

Criterio 4. La infraestructura cuenta con un estudio técnico o un proyecto redactado: tomando como referencia lo planteado en el criterio 3 , sucede lo mismo con aquellas infraestructuras turísticas que tengan un proyecto redactado. Ello permite que su licitación sea prácticamente inmediata en caso de que tenga financiación. Asimismo, supone disponer de una unidad de inversión. Del mismo modo, da cuenta de la decidida voluntad y una vocación ejecutiva de la institución proponente acerca de la necesidad de disponer de tal infraestructura.

Criterio 5. La infraestructura dispone de financiación previa: el hecho que de un porcentaje del coste total de las infraestructuras turísticas cuente con financiación previa constituye un criterio de priorización. Así, el PITCAN se plantea como una fórmula para completar dicha financiación, con el fin de viabilizar la materialización de la misma. No obstante, el bajo porcentaje de respuesta del campo relativo al porcentaje y origen (externo o propia) de la financiación determinó la conveniencia de no tenerlo en cuenta, al no ser representativa la muestra; lo mismo pasó con la cuantía del proyecto.

Criterio 6. Grado de ejecución de la infraestructura turística: el hecho de que una infraestructura propuesta esté licitada o en ejecución da cuenta 
Actas del Seminario Internacional Destinos Turísticos Inteligentes:

nuevos horizontes en la investigación y gestión del turismo

Universidad de Alicante, 26 y 27 de octubre de 2017

del interés e importancia de la entidad promotora sobre la misma. En estos casos, como sucede con el criterio 4, ambos estados dan cuenta de la decidida voluntad de la institución proponente acerca de la necesidad de disponer de la infraestructura.

\section{Criterio 7. La infraestructura ha sido propuesta por parte de más de una} entidad: el hecho de que más de una institución proponga una misma infraestructura da cuenta de su importancia estratégica, al resultar coherente con las expectativas urgentes de determinados actores turísticos. $\mathrm{Si}$ bien en algunos casos resulta de una cierta "casualidad», en otros casos, esta solicitud coincidente es resultado de un ejercicio diálogo, acuerdo y concertación en torno a la necesidad y prioridad de la infraestructura en cuestión.

Criterio 8. La infraestructura está contemplada en un Plan de Modernización, Mejora e Incremento de la Competitividad: los Planes de Modernización, Mejora e Incremento de la Competitividad (PMM) aspiran a convertirse en instrumentos de ordenación y gestión urbanística dirigidos a viabilizar y agilizar la ejecución de los proyectos de renovación de los espacios privados (las infraestructuras turísticas de alojamiento y complementarias) y privados (alojamientos y equipamientos) de las áreas turísticas maduras. Constituyen soluciones pioneras, novedosas y singulares, en cuanto se plantean como planes ad hoc. Su redacción y tramitación se encargó a la empresa pública GesPlan, con la única excepción del PMM de Puerto de la Cruz, que ha sido redactado por la empresa pública Gestur Tenerife. Los PMM abarcan un $86,7 \%$ de las plazas turísticas de Canarias.

La ponderación de una infraestructura turística que está presente en los PMM otorga la importancia (territorial, económica social, etc.) que tienen las áreas turísticas «maduras», "consolidadas», "con experiencia» o «pioneras» de litoral. Para ello se han recopilado las 445 actuaciones previstas en los 20 PMM aprobados, suspendidos y en tramitación, las cuales se han cruzado con las propuestas por las entidades insulares y municipales.

Finalmente, el modelo de definición de la importancia estratégica de las infraestructuras se desarrolla matricialmente, completando las casillas de cruce en función de los criterios y ponderaciones del cuadro 1. 
Actas del Seminario Internacional Destinos Turísticos Inteligentes: nuevos horizontes en la investigación y gestión del turismo

Universidad de Alicante, 26 y 27 de octubre de 2017

\begin{tabular}{|c|c|c|c|c|}
\hline \multicolumn{4}{|c|}{$\begin{array}{l}\text { Cuadro1. Criterios de valoración de la importancia } \\
\text { estratégica }\end{array}$} & \multirow[b]{2}{*}{$\begin{array}{l}\text { Peso del } \\
\text { criterio } \\
\text { sobre el } \\
\text { toral (en \%) }\end{array}$} \\
\hline & Criterios de valoración & Ponderaciones & & \\
\hline \multirow{4}{*}{ Criterio 1} & \multirow{4}{*}{ Aspectos multidisciplinares } & $\begin{array}{l}\text { Adecuación de la infraestructura a la estra } \\
\text { marketing insular }\end{array}$ & tegia de 15 & \multirow{4}{*}{$\leq 50$} \\
\hline & & $\begin{array}{l}\text { Importancia de las infraestructuras para e } \\
\text { turístico insular }\end{array}$ & I modelo & \\
\hline & & Afluencia y opinión de los visitantes & 10 & \\
\hline & & Impacto económico de la infraestructura & 10 & \\
\hline \multirow{3}{*}{ Criterio 2} & \multirow{3}{*}{$\begin{array}{l}\text { Relevancia estratégica } \\
\text { otorgada por el equipo } \\
\text { externo (consulta a } \\
\text { técnicos, responsables } \\
\text { públicos y profesionales) }\end{array}$} & Valor 1 [bajo] & Hasta 5 & \multirow{3}{*}{$\leq 20$} \\
\hline & & Valor 2 [medio] & $\begin{array}{l}\text { Hasta } \\
10\end{array}$ & \\
\hline & & Valor 3 [alto] & $\begin{array}{l}\text { Hasta } \\
20\end{array}$ & \\
\hline \multirow{2}{*}{ Criterio 3} & \multirow{2}{*}{$\begin{array}{l}\text { Inclusión de la } \\
\text { infraestructura en un plan o } \\
\text { programa }\end{array}$} & No & 0 & \multirow[b]{2}{*}{$\leq 5$} \\
\hline & & Sí & 5 & \\
\hline \multirow{2}{*}{ Criterio 4} & \multirow{2}{*}{$\begin{array}{l}\text { La infraestructura cuenta } \\
\text { con un estudio técnico o } \\
\text { proyecto redactado }\end{array}$} & No & 0 & \multirow{2}{*}{$\leq 5$} \\
\hline & & Sí & 5 & \\
\hline \multirow{2}{*}{ Criterio 5} & \multirow{2}{*}{ Tiene financiación } & No & 0 & \multirow{2}{*}{$\leq 5$} \\
\hline & & Sí & 5 & \\
\hline \multirow{3}{*}{ Criterio 6} & \multirow{3}{*}{ Grado de ejecución } & Sin ejecutar & 0 & \multirow{3}{*}{$\leq 5$} \\
\hline & & Licitada & 2,5 & \\
\hline & & En ejecución & 5 & \\
\hline \multirow{2}{*}{ Criterio 7} & \multirow{2}{*}{$\begin{array}{l}\text { Ha sido propuesta por más } \\
\text { de una entidad }\end{array}$} & No & 0 & \multirow{2}{*}{$\leq 5$} \\
\hline & & Sí & 5 & \\
\hline \multirow[b]{2}{*}{ Criterio 8} & \multirow{2}{*}{$\begin{array}{l}\text { Está contemplada en un } \\
\text { Plan de } \\
\text { Modernización, Mejora e } \\
\text { Incremento de la } \\
\text { Competitividad }\end{array}$} & No & 0 & \multirow[b]{2}{*}{$\leq 5$} \\
\hline & & Sí & 5 & \\
\hline
\end{tabular}

Elaboración propia

Un ejemplo de valoración es el siguiente: 
Actas del Seminario Internacional Destinos Turísticos Inteligentes:

nuevos horizontes en la investigación y gestión del turismo

Universidad de Alicante, 26 y 27 de octubre de 2017

\begin{tabular}{|c|c|c|c|c|c|c|c|c|c|c|c|c|c|}
\hline $\begin{array}{l}\text { Número de } \\
\text { orden en } \\
\text { relevancia } \\
\text { estratégica }\end{array}$ & Nombre & Isla & Municipio & $\begin{array}{c}\text { Entidades } \\
\text { proponentes }\end{array}$ & $\frac{\overline{2}}{3}$ & 竭 & 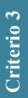 & $\frac{7}{0}$ & 象 & : & 音 & 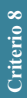 & Valoración \\
\hline \multirow[b]{2}{*}{1} & \multirow{2}{*}{$\begin{array}{c}\text { Mejora } \\
\text { de la } \\
\text { eficiencia } \\
\text { del } \\
\text { complejo } \\
\text { Costa } \\
\text { Martiánez }\end{array}$} & \multirow[b]{2}{*}{ Tenerife } & \multirow[b]{2}{*}{$\begin{array}{l}\text { Puerto de } \\
\text { La Cruz }\end{array}$} & $\begin{array}{c}\text { Ayuntamiento de } \\
\text { Puerto de La } \\
\text { Cruz }\end{array}$ & \multirow[b]{2}{*}{34,5} & \multirow[b]{2}{*}{15} & \multirow[b]{2}{*}{5} & \multirow[b]{2}{*}{5} & \multirow[b]{2}{*}{5} & \multirow[b]{2}{*}{5} & \multirow[b]{2}{*}{5} & \multirow[b]{2}{*}{5} & \multirow[b]{2}{*}{74,1} \\
\hline & & & & $\begin{array}{l}\text { Consorcio de } \\
\text { Rehabilitación } \\
\text { Urbanística de } \\
\text { Puerto de La } \\
\text { Cruz }\end{array}$ & & & & & & & & & \\
\hline
\end{tabular}

El resultado del proceso fue la catalogación y priorización de 632 infraestructuras turísticas. Estas fueron propuestas por 37 entidades: el Gobierno de Canarias, Promotur, los siete Cabildos Insulares, 24 municipios (el 27\%), la Asociación empresarios turísticos de Fuerteventura, una entidad privada (Gema Arquitectos) y el grupo de investigación universitario del Patrimonio (Departamento de Construcción Arquitectónica de la Universidad de Las Palmas de Gran Canaria). Asimismo, Gestión y Planeamiento Territorial y Medioambiental, S.A (Gesplan) se ha incorporado, en cuanto es la empresa pública es la encargada de los Planes de Modernización, Mejora e Incremento de la Competitividad.

\section{Conclusiones}

Las infraestructuras turísticas, entendidas en tanto que dotaciones, equipamientos, instalaciones o, incluso, servicios ofrecidos en ellos, son básicas para el adecuado funcionamiento de los destinos. Sin embargo, en ocasiones no se desarrollan de manera integrada y sistemática, sino atendiendo, más bien, a determinados intereses o perspectivas parciales. Desde el paradigma de la gestión inteligente de destinos turísticos, el PITCAN se ha desarrollado mediante una metodología de identificación, selección, jerarquización, valoración y priorización de infraestructuras en base a un proceso abierto a la participación y un sistema de indicadores ponderados que combinan la perspectiva de oferta y demanda.

Se trata de poner la tecnología y los datos al servicio de la toma inteligente de decisiones y fomentar la participación de diferentes agentes en los procesos de planificación y gestión orientados a la mejora del destino, no sólo teniendo en cuenta las demandas, expectativas y experiencias de los 
Actas del Seminario Internacional Destinos Turísticos Inteligentes:

nuevos horizontes en la investigación y gestión del turismo

Universidad de Alicante, 26 y 27 de octubre de 2017

turistas actuales, sino también la perspectiva estratégica de instituciones y otros actores del destino.

Por otra parte, el PITCAN aspira a convertirse en un cauce formal, directo, activo, ágil y eficiente de concertación público-privada, acorde a la propia transversalidad del sector turístico, con los que no sólo dinamizar el proceso de adecuación de las infraestructuras turísticas a la demanda y al correcto desarrollo de los productos turísticos, sino también impulsar la innovación en la gestión del destino, desechando los modelos tradicionales, ampliamente cuestionados. Asimismo, se ha concebido como un plan abierto a la interacción ágil y mejora continua que tanto puede ser modificado en estos años de vigencia como constituye una excelente base para su reinterpretación más allá de este periodo.

Entre las limitaciones pueden destacarse que algunos de los agentes consultados decidieron no participar proponiendo o valorando infraestructuras, así como la inexistencia o imposibilidad de acceso a datos relativos a los perfiles de turista y actividades realizadas por estos en alguna de las escalas planteadas en el proyecto.

En definitiva, se plantea una metodología para la toma inteligente de decisiones en destinos turísticos, en este caso, en materia de gestión de infraestructuras turísticas. Esta tiene potencial para ser adaptada y aplicada en otros contextos, así como a problemáticas similares.

\section{Referencias}

Boes, K., Buhalis, D., and Inversini, A. (2015) «Conceptualising Smart Tourism Destination Dimensions», En Tussyadiah, I., and Inversini, A., (eds), ENTER 2015 Proceedings, Lugano, Springer-Verlag, Wien

Buhalis, D. \& Amaranggana, A. (2014) «Smart Tourism Destinations», En Xiang, Z., Tussyadiah, I., (eds) Information and Communication Technologies in Tourism 2014, pp.553-564

Buhalis, D. \& Amaranggana, A. (2015). «Smart Tourism Destinations Enhancing Tourism Experience through Personalisation of Services», En Tussyadiah, I., and Inversini, A., (eds), ENTER 2015 Proceedings, Lugano, Springer-Verlag, Wien

Caragliu, Andrea., Del Bo, C., \& Nijkam p, P. (2009). "Smart cities in Europe». 3rd Central European Conference in Regional Science

Ivars Baidal, J.; F. Solsona Monzonís, J. y Giner Sánchez, D. (2016). Gestión turística y tecnologías de la información y la comunicación (TIC): El nuevo enfoque de los destinos inteligentes, Documents d'Anàlisi Geogràfica vol. 62/2, pp. 327-346. 
Actas del Seminario Internacional Destinos Turísticos Inteligentes:

nuevos horizontes en la investigación y gestión del turismo

Universidad de Alicante, 26 y 27 de octubre de 2017

SEGITTUR (2013): Destinos turísticos inteligentes. URL: http://www.segittur.es/ opencms/export/sites/segitur/.content/galerias/descargas/documentos/Presentacin-Destinos-Tursticos-Inteligentes.pdf.

Vera Rebollo, J.F.; López Palomeque, Marchena, M. y Antón Clavé, S. (2011). «Análisis territorial del turismo y planificación de destinos turísticos». Valencia, Tirant lo Blanch.

Wang, D.; Li, X. \& Yunpeng, L. (2013). «China's smart tourism destination iniciative: A taste of the service-dominant logic». Journal of Destination Marketing \& Management. Vol. 2: 59-61 